\title{
CASE REPORT \\ Can FES-rowing mediate bone mineral density in SCI: a pilot study
}

\author{
RS Gibbons ${ }^{1}$, ID McCarthy ${ }^{2}$, A Gall ${ }^{2,3}$, CG Stock ${ }^{1}$, J Shippen ${ }^{4}$ and BJ Andrews ${ }^{5}$
}

\begin{abstract}
Study design: A single case study.
Objectives: To compare proximal tibia trabecular bone mineral density (BMD) of a participant with complete spinal cord injury (SCI), long-termed functional electrical stimulation-rowing (FES-R) trained, with previously reported $\mathrm{SCl}$ and non-SCl group norms. To estimate lower limb joint contact forces (JCFs) in the FES-R trained participant.
\end{abstract}

Setting: UK University and orthopaedic hospital research centre.

Methods: Bilateral proximal tibial trabecular BMD of the FES-R trained participant was measured using peripheral quantitative computerised tomography, and the data were compared with $\mathrm{SCl}$ and non-SCl groups. An instrumented four-channel FES-R system was used to measure the lower limb JCFs in the FES-R trained participant.

Results: Structurally, proximal tibial trabecular BMD was higher in the FES-R trained participant compared with the SCI group, but was less than the non-SCl group. Furthermore, left $\left(184.7 \mathrm{mg} \mathrm{cm}^{-3}\right)$ and right $\left(160.7 \mathrm{mg} \mathrm{cm}^{-3}\right)$ BMD were well above the threshold associated with non-traumatic fracture. The knee JCFs were above the threshold known to mediate BMD in SCI, but below threshold at the hip and ankle.

Conclusion: As pathological fractures predominate in the distal femur and proximal tibia in chronic SCl patients, the fact that the FES-R trained participant's knee JCFs were above those known to partially prevent bone loss, suggests that FES-R training may provide therapeutic benefit. Although the elevated bilateral proximal tibial BMD of the FES-R participant provides circumstantial evidence of osteogenesis, this single case precludes any statement on the clinical significance. Further investigations are required involving larger numbers and additional channels of FES to increase loading at the hip and ankle.

Spinal Cord (2014) 52, S4-S5; doi:10.1038/sc.2014.112

\section{INTRODUCTION}

Osteoporosis is a known secondary complication of spinal cord injury (SCI) characterised by low bone mineral density (BMD), which results in a high incidence of pathological fractures in the distal femur and proximal tibia. ${ }^{1}$ Physical therapy is one strategy under investigation to mediate bone loss in SCI by increasing muscle-induced skeletal loading. ${ }^{2}$ In the SCI model, functional electrical stimulation (FES) is required to activate paralysed muscle. Although the dose-response in the SCI model is unknown, compressive loads of 1-2 times body weight, delivered by FES-induced muscle contractions, have been found to partially prevent bone loss after SCI. ${ }^{2}$ The aims of this study were to: (1) compare the lower limb BMD of a participant with long-term FESrowing (FES-R) trained with SCI and non-SCI groups, using peripheral quantitative computerised tomography imaging and (2) establish the magnitude of joint contact forces (JCFs) in the lower limbs of the FES$\mathrm{R}$ trained participant.

\section{MATERIALS AND METHODS}

The participant for these trials (Table 1), and author of this paper, had been FES-R trained for $>8$ years as described in reference 3 using quadriceps and hamstring controlled FES-activation. The FES-R system was originally developed by our group and is described elsewhere. ${ }^{3}$ BMD measurements were performed using peripheral quantitative computerised tomography employing protocols from a previous study. ${ }^{4}$ The FES-R machine was instrumented to measure force data in the handle and foot stretchers. Kinematics and kinetics of the rowing action were recorded using a Vicon MX40 motion analysis system (Vicon, Oxford, UK). JCFs were calculated using a biomechanical modelling package (Biomechanics of Body, Coventry University, Coventry, UK).

\section{RESULTS}

The bilateral proximal tibial trabecular BMD in the FES-R trained participant was greater when compared with the mean value of the SCI group, but less than the non-SCI group (Table 1). ${ }^{4}$ The left proximal tibial trabecular BMD was found to be $\sim 15 \%$ greater than the right in this participant (Table 2). Since participating in FES-R training, this participant's routine dual-energy X-ray absorptiometry hip scores have also increased by $\sim 15 \%$, with the T-score increasing from -2.3 to -1.7 .

The participant's knee JCFs, normalised to body weight, were greater than the JCF known to mediate BMD loss in SCI. ${ }^{2}$ However, hip and ankle forces were lower (Figure 1). Interestingly, the left knee JCF was $\sim 25 \%$ higher than the right in this participant, perhaps accounting for the higher proximal BMD in the left tibia.

${ }^{1}$ Centre for Sports Medicine and Human Performance, Brunel University, London, UK; ${ }^{2}$ UCL Institute of Orthopaedics and Musculoskeletal Science, Stanmore, UK; ${ }^{3}$ London Spinal Cord Injury Centre, Royal National Orthopaedic Hospital, Stanmore, UK; ${ }^{4}$ Department of Industrial Design, Coventry University, Coventry, UK and ${ }^{5}$ Nuffield Department of Surgical Sciences, Oxford University, Oxford, UK

Correspondence: RS Gibbons, Centre for Sports Medicine and Human Performance, Brunel University, Kingston Lane, Uxbridge, London UB8 3PH, UK.

E-mail: robin.gibbons@brunel.ac.uk

Received 18 March 2014; revised 17 April 2014; accepted 26 April 2014 
Table 1 Long-term FES-rowing-trained participant characteristics compared with untrained chronic $\mathrm{SCl}$ and non-SCI groups

\begin{tabular}{lcccc}
\hline & Age (years) & TPI (years) & SCl & AlS \\
\hline FES rower & 58 & 13.5 & T4 & A \\
& & & & \\
$\begin{array}{l}\text { Chronic SCl group } \\
\quad \text { Mean } \pm \text { s.d. }\end{array}$ & $32 \pm 9$ & $6.6 \pm 2.8$ & & \\
$\quad$ Range & $25-43$ & $4.1-12.6$ & C6-T10 & A \\
Non-SCl group & & & & \\
$\quad$ Mean \pm s.d. & $37 \pm 9$ & $\mathrm{n} / \mathrm{a}$ & & \\
Range & $22-55$ & $\mathrm{n} / \mathrm{a}$ & $\mathrm{n} / \mathrm{a}$ & $\mathrm{n} / \mathrm{a}$ \\
\hline
\end{tabular}

Abbreviations: A, complete SCI; AIS, American Spinal Injury Association Impairment Scale; C, cervical; FES, functional electrical stimulation; $\mathrm{SCl}$, spinal cord injury; T, thoracic; TPI, time post injury.

Note that before injury, the FES rower's habitual exercise training consisted of weekly highvolume swimming training. Post injury and before FES-R training this exercise regime continued concurrent with upper body weight training and daily static standing. There is therefore no evidence to suggest this participant would have above average trabecular bone mineral density in his lower limbs.

Table 2 Proximal tibial trabecular bone mineral density of the long-term FES-rowing-trained participant compared with untrained chronic $\mathrm{SCl}$ and non-SCl groups

\begin{tabular}{|c|c|c|c|c|}
\hline & \multicolumn{2}{|c|}{ FES rower } & \multirow{2}{*}{$\begin{array}{l}\text { Chronic SCl } \\
\text { group }(\mathrm{n}=9)\end{array}$} & \multirow{2}{*}{$\begin{array}{c}\text { Non-SCl group } \\
\quad(\mathrm{n}=14)\end{array}$} \\
\hline & Left & Right & & \\
\hline $\begin{array}{l}\text { Proximal tibia trabecular } \\
\text { density }\left(\mathrm{mg} \mathrm{cm}^{-3}\right)\end{array}$ & 184.7 & 160.7 & $131.5 \pm 51.1$ & $212.8 \pm 48.9$ \\
\hline
\end{tabular}

Abbreviations: FES, functional electrical stimulation; $\mathrm{SCl}$, spinal cord injury.

Data are mean and where indicated \pm 1 s.d. Note, the BMD values in the FES-rowing trained participant are higher than that in the chronic SCl group, and the left BMD in this participant is greater than the right. The average is within 1 s.d. of the mean for the non-SCl group.

\section{DISCUSSION}

We aimed to compare the bilateral proximal tibial trabecular BMD in an FES-R trained participant with untrained SCI and non-SCI groups. The fact that the FES-R trained participant had greater values than the SCI group may have clinical significance, as the highest incidence of pathological fractures in SCI occur in the distal femur and proximal tibia. ${ }^{1}$ Furthermore, the higher knee BMD values in this participant may be explained by the increased JCFs as a result of FES-R training. The asymmetric knee JCFs appear to correspond to the greater BMD values in this participant, which provides evidence of a relationship between the magnitude of mechanical loading and changes in the structure of the bones affected.

It has been hypothesised that muscle-induced loading (or unloading) is one factor that stimulates changes in the structural properties of weight-bearing bones. ${ }^{5}$ The underlying mechanisms that control these changes may still function in SCI when using FES-assisted muscle-induced loading on a site-specific basis. ${ }^{2}$ The elevated knee $\mathrm{BMD}$, relative to the hip and ankle BMD in the FES-R trained participant, is in line with these theories. Moreover, as the knee JCFs in the FES-R trained participant are greater than the known tibial fracture threshold in SCI, ${ }^{1}$ the rate of bone adaptation appears to be sufficient to accommodate the increased limb loading during long-term FES-R training. Interestingly, although of lower magnitude, the muscle-inactive hip joint loading appears sufficient to improve the two-dimensional hip scores in this participant (Figure 1).

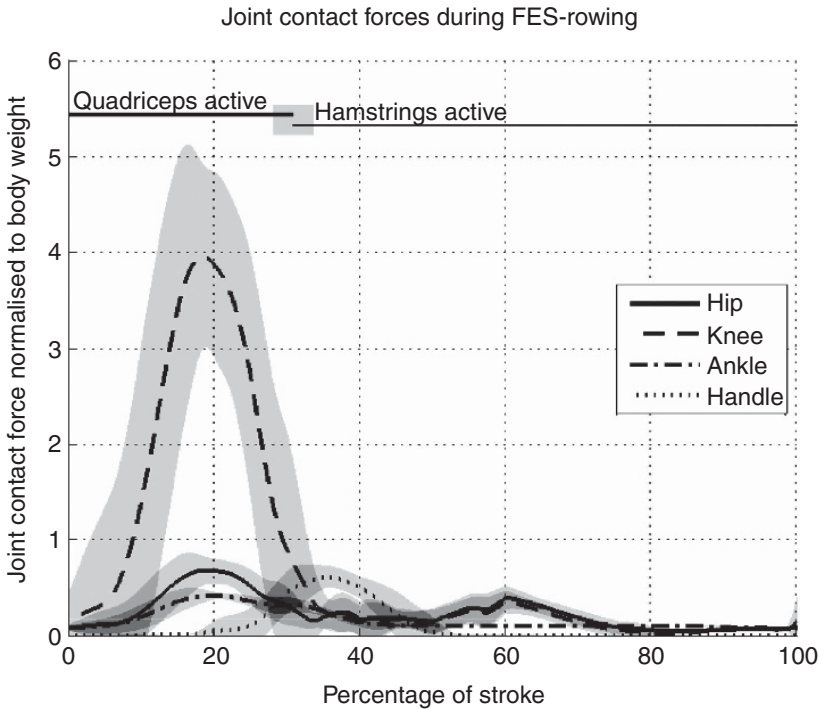

Figure 1 Mean JCFs of the FES-R trained participant normalised to body weight. Data are mean $\pm 1 \mathrm{~s}$.d. (shaded areas). Horizontal lines denote the quadriceps and hamstring FES-active phases. Note, the knee JCF in this participant is higher than the hip or ankle. Also shown is the rowing ergometer handle pull force.

Given the limitations of this single-case preliminary report, the clinical significance of these findings is that FES-R may provide a practical form of patterned cyclical loading sufficient to mediate BMD in long bones loaded by FES-induced muscle force actions. There have been no reported skeletal complications attributed to FES-R training in over 20 participants over several years. It is therefore reasonable to hypothesise that fracture risk is mediated with the present FES-R training protocols. As pathological fractures in the distal femur and proximal tibia predominate in SCI, FES-R may improve the bone health in addition to the cardiovascular benefits previously reported. ${ }^{3}$ Further clinical investigations are suggested involving larger numbers and additional channels of FES to increase loading at the hip and ankle.

\section{DATA ARCHIVING}

There were no data to deposit.

\section{CONFLICT OF INTEREST}

The authors declare no conflict of interest.

\section{ACKNOWLEDGEMENTS}

We are grateful to the INSPIRE Foundation for funding this work.

1 Zehnder Y, Luthi M, Michel D, Knecht H, Perrelet R, Neto I et al. Long-term changes in bone metabolism, bone mineral density, quantitative ultrasound parameters, and fracture incidence sfter spinal cord injury: a cross-sectional observational study in 100 paraplegic men. Osteoporosis Int 2004; 15: 180-189.

2 Shields RK, Dudley-Janoroski S, Frey Law LA. Electrically induced muscle contractions influence bone density decline after spinal cord injury. Spine 2006; 31: 548-553.

3 Hettinga DM, Andrews BJ. The feasibility of functional electrical stimulation indoor rowing for high-energy training and sport. Neuromodulation 2007; 10: 291-297.

4 McCarthy ID, Bloomer Z, Gall A, Keen R, Ferguson-Pell M. Changes in the structural and material properties of the tibia in patients with spinal cord injury. Spinal Cord 2012; 50: 333-337.

5 Frost HM. Bone's mechanostat: a 2003 update. Anat Rec A Discov Mol Cell Evol Biol 2003; 275: 1081-1101. 